\title{
Los "doce roles del docente de medicina": un estudio piloto de tres universidades públicas de Paraguay
}

\author{
Julio Torales ${ }^{1}$, Hugo Gustavo Kunzle-Elizeche ${ }^{2}$, Iván Barrios ${ }^{1}$, Carlos Miguel Rios-González ${ }^{3}$, Albert \\ Rafael Barrail' ${ }^{2}$, Israel González-Urbieta ${ }^{1}$, Raúl Real ${ }^{4}$
}

\begin{abstract}
${ }^{1}$ Universidad Nacional de Asunción, Facultad de Ciencias Médicas. San Lorenzo, Paraguay Universidad Nacional del Este, Facultad de Ciencias de la Salud. Minga Guazú, Paraguay ${ }^{3}$ Universidad Nacional de Caaguazú, Facultad de Ciencias Médicas. Coronel Oviedo, Paraguay

${ }^{4}$ Universidad Nacional de Itapúa, Facultad de Medicina. Encarnación, Paraguay
\end{abstract}

Cómo referenciar este artículo/ How to reference this article:
Torales J, Kunzle-Elizeche HG, Barrios I, RiosGonzález CM, Barrail AR, González-Urbieta I, et al. Los "doce roles del docente de medicina": un estudio piloto de tres universidades públicas de Paraguay. Mem. Inst. Investig. Cienc. Salud. 2018; 16(2): 55-64

\section{R E S U M E N}

Reconocer los múltiples roles que tienen los docentes de medicina es importante para prepararlos para la compleja tarea de enseñar la ciencia médica. El objetivo de este trabajo fue determinar la importancia que dan los docentes de medicina de 3 universidades públicas de Paraguay a los "Doce roles de un docente de medicina" planteados por Harden y Crosby. Fue un estudio observacional, descriptivo, de corte transversal, con muestreo por conveniencia. Los participantes fueron docentes de Medicina, de la Universidad Nacional de Asunción, Nacional del Este y Nacional de Itapúa, con al menos 2 años de experiencia, que completaron un cuestionario que midió la importancia que dan a los doce roles del docente de medicina. Para ese fin, se utilizó una versión en español de la teoría de Harden y Crosby, traducida con autorización de sus autores. Participaron del estudio 73 docentes. La mayoría percibió su papel más importante como proveedor de información en las prácticas o en la clínica $(4,68 \pm 0,57)$, como modelo de rol en el ámbito laboral $(4,53 \pm 0,60)$ y en la enseñanza $(4,45 \pm 0,67)$, y como planificador o participante en las evaluaciones del estudiante $(4,41 \pm 0,62)$. Estos resultados nos permiten afirmar que el docente de medicina de Paraguay se ve a sí mismo como más que un instructor de clases teóricas o prácticas clínicas, sino cumpliendo muchos diferentes roles al mismo tiempo. Los docentes de medicina pueden desempeñar un papel importante en la realización de estudios de investigación pedagógica que permitan mejorar las prácticas de enseñanza y aprendizaje de la medicina.

Palabras clave: roles docentes, medicina, educación médica.

\section{The "twelve roles of the medical teacher": a pilot study of three public universities of Paraguay}

\section{A B S T R A C T}

Recognizing the multiple roles that medical teachers have is important to prepare them for the complex task of teaching medical sciences. The objective of this study was to determine the importance that medicine teachers from three public universities in Paraguay give to the "Twelve roles of a medical teacher" proposed by Harden and Crosby. This was an observational descriptive cross-sectional study with convenience sampling. The participants were teachers of Medicine at the Universidad Nacional de Asunción, Universidad Nacional del Este and Universidad Nacional de Itapúa, with at least 2 years of experience, who completed a questionnaire that measured the importance they give to the twelve roles of the medical teacher. For that purpose, a Spanish version of the Harden and Crosby theory was used and translated with the authorization of its authors. Seventy three teachers participated in the study. The majority perceived its most important role as a provider of information in the clinical rounds or in the clinic $(4.68 \pm 0.57)$, as a role model in the 
workplace (4.53 \pm 0.60$)$ and in teaching $(4.45 \pm 0.67)$, and as a planner or participant in the student evaluations $(4.41 \pm 0.62)$. These results allow us to affirm that the medical teacher of Paraguay sees himself more as an instructor of theoretical classes or clinical practices, but they see themselves as fulfilling many different roles at the same time. Medical teachers can play an important role in conducting pedagogical research studies to improve the practices used for teaching and medicine.

Keywords: teaching roles, medicine, medical education.

\section{INTRODUCCIÓN}

Actualmente, la educación médica se ve en la necesidad de formar profesionales de la salud con capacidad para poder aplicar las herramientas aprendidas durante el grado y el postgrado a la práctica cotidiana y solucionar los problemas de salud de la población. Para satisfacer esa necesidad, y lograr así impartir una docencia de calidad, es crucial la formación en Pedagogía ${ }^{(1,2)}$ y reconocer los múltiples roles que puede ejercer un docente de medicina.

La educación médica superior se enfrenta a múltiples desafíos como la globalización, la expansión y excesiva disponibilidad de información, y la mayor demanda social para una formación más integral y pertinente. Por todo esto, un docente de medicina, además de su formación médica, debe poseer formación específica en educación, teniendo en cuenta que el académico universitario puede asumir varios roles: investigador, coordinador, docente, evaluador, entre otros ${ }^{(3-5)}$.

Una cuestión clave es definir cuál sería el papel que tiene el docente en el contexto de los rápidos cambios que se han y siguen suscitando en la educación médica. Esta interrogante fue abordada por Ronald Harden y Joy Crosby, del Centro de Educación Médica de la Universidad de Dundee (Escocia), que sugirieron los doce roles de un docente de medicina. Estos autores afirman que a menos que los docentes no acuerden los roles que deben cumplir, no se podrá seleccionar a las mejoras personas para los puestos de enseñanza, no se podrá definir qué es "enseñar bien" y no se podrán instalar sistemas de promoción o reconocimiento docente ${ }^{(6,7)}$.

Para definir los roles de un docente de medicina, Harden y Crosby, desarrollaron un modelo a través de tres fuentes: a. Un análisis de las tareas que se esperan sean cumplidas por un docente en el diseño e implementación del currículo de una escuela de medicina ${ }^{(8)}$; b. Un estudio de los diarios llevados por 12 estudiantes de medicina, durante un periodo de 3 meses, y un análisis de sus comentarios relacionados a los roles del docente; y, c. Un análisis de la teoría y la literatura existente sobre el particular. Finalmente, el modelo de Harden y Crosby, quedó establecido en 6 áreas principales de la actividad docente, cada una de ellas con 2 roles (Tabla 1 ).

Tabla 1. Modelo de Harden y Crosby

\begin{tabular}{ll}
\hline \multicolumn{1}{c}{ Área } & Roles \\
\hline El docente como & 1. Docente en clases teóricas. \\
proveedor de información & 2. Docente en las prácticas o en la clínica. \\
El docente como modelo de rol & $\begin{array}{l}\text { 3. Modelo de rol en el ámbito laboral. } \\
\text { 4. Modelo de rol en el ámbito de la enseñanza. }\end{array}$ \\
El docente como facilitador & 5. Mentor, asesor o tutor del estudiante. \\
El docente como examinador & 6. Facilitador del aprendizaje. \\
& 7. Planificador o participante en las evaluaciones del \\
El docente como planificador & 8. Evaluador del currículo o de los planes de enseñanza. \\
El docente como & 10. Organizador del curso. \\
desarrollador de recursos & 11. Productor de guías de estudio. \\
\hline
\end{tabular}


Los roles antedichos fueron validados por 251 docentes de la Universidad de Dundee, Escocia, y fueron aprobados, además, por la Asociación para la Educación Médica en Europa (AMEE, por sus siglas en inglés), la que los incluyó formalmente en la Guía de Trabajo Nº 20 de la asociación en $2000^{(7)}$. A partir de ahí, numerosas investigaciones en educación médica, en todo el mundo, han utilizado estos roles docentes.

Con base en lo anterior, el objetivo del presente trabajo fue determinar la importancia que dan los docentes de medicina de la Universidad Nacional de Asunción, la Universidad Nacional del Este y la Universidad Nacional de Itapúa, todas universidades públicas de Paraguay, a los doce roles planteados en la teoría de Harden y Crosby.

\section{METODOLOGÍA}

\section{Diseño}

Estudio observacional, descriptivo, de corte transversal, con muestreo por conveniencia, realizado entre los meses de noviembre y diciembre de 2017.

\section{Población en estudio y recolección de datos}

Los participantes del estudio fueron docentes de medicina de la Universidad Nacional de Asunción, la Universidad Nacional de Este y la Universidad Nacional de Itapúa, con al menos 2 años de experiencia en docencia y que pertenezcan a una sola de las Universidades incluidas. A todos los participantes se les invitó a completar un cuestionario, mediante correo electrónico y a través del servicio de mensajería WhatsApp. Esta invitación permitió, además, informar los objetivos propuestos por el estudio. Se excluyeron a aquellos docentes que no completaron la totalidad de la encuesta. Finalmente, la muestra quedó constituida por 73 participantes, siendo este un estudio piloto. Todos los participantes tenían al español como lengua materna, idioma en el que estaba escrito el cuestionario.

\section{Cuestionario}

El cuestionario fue elaborado mediante la herramienta Google Drive $\subseteq$, y estaba conformado por 3 secciones: la primera, que contenía el consentimiento informado, la segunda, sobre variables socio-académicas de los participantes (sexo, edad, años de docencia, y Universidad donde enseña) y la tercera, que midió la importancia que dan los participantes a los doce roles del docente de medicina. Para ese fin, se utilizó una versión en español de la teoría de Harden y $\mathrm{Crosby}^{(7)}$, traducida al español con autorización de sus autores. Previamente, esta encuesta fue validada por la opinión experta de 5 docentes de medicina, con especialización o maestría en docencia médica. Las preguntas sobre la teoría de Harden y Crosby están distribuidas en 6 áreas, con 6 roles cada uno (12 en total), donde se indaga sobre la importancia que el docente de medicina da a cada uno de los mismos. Esta valoración de la importancia puede ir desde "ninguna" a "mucha" y sus valores van del "1" al "5" (donde 5 representa la mayor importancia valorada para un rol).

\section{Análisis estadístico}

Luego del control de calidad de los datos proveídos por el cuestionario electrónico, las variables fueron exportadas y analizadas con el paquete estadístico Epi Info versión 7.2 (Centers for Disease Control and Prevention, Atlanta, Estados Unidos de América). Se utilizó estadística descriptiva para resumir los datos, expresándose los resultados como medidas de tendencia central, dispersión y tablas de frecuencias.

\section{Asuntos éticos}

El protocolo de investigación fue aprobado por el Comité de Ética de la Investigación de la Facultad de Ciencias Médicas de la Universidad Nacional de Asunción. La participación del estudio fue libre y voluntaria y los participantes firmaron un consentimiento informado, que se encontraba en la primera parte del cuestionario electrónico. Se cumplieron con los principios éticos establecidos en la Declaración de Helsinki de la Asociación Médica Mundial, relativos a la investigación en seres humanos.

\section{RESULTADOS}

Del presente estudio participaron 73 profesores de la Universidad Nacional de Asunción, Universidad Nacional del Este y Universidad Nacional de Itapúa, con edades comprendidas entre 29 y 65 años y una media de $45 \pm 10$ años. El 56\% de los participantes era del sexo masculino y el $42,5 \%$ enseñaba en la Universidad Nacional del Este, $41,1 \%$ en la Universidad Nacional de Asunción y el resto en la Universidad Nacional de Itapúa. La antigüedad promedio como docente de los sujetos estudiados fue de $10 \pm 7$ años. 
En cuanto a los resultados de los roles docentes y su importancia, los datos cuantitativos pueden verse en la tabla 2 . La consistencia interna del cuestionario, medida por el alfa de Cronbach, resultó alta con $a=0,91$.

Tabla 2. Distribución de medias (y desviaciones estándar) de la importancia otorgada por los participantes a los doce roles docentes $(n=73)$.

\section{Rol docente}

\section{El docente como proveedor de información:}

a. Docente en clases teóricas.

b. Docente en las prácticas o en la clínica.

2. El docente como modelo de rol:

a. Modelo de rol en el ámbito laboral.

b. Modelo de rol en el ámbito de la enseñanza.

3. El docente como facilitador:

a. Mentor, asesor o tutor del estudiante.

b. Facilitador del aprendizaje.

4. El docente como examinador:

a. Planificador o participante en las evaluaciones del estudiante.

b. Evaluador del currículo o de los planes de enseñanza.

5. El docente como planificador:

a. Planificador del currículo.

b. Organizador del curso.

6. El docente como desarrollador de recursos:

a. Productor de guías de estudio.

b. Desarrollador de materiales educativos.
Media DE

3,99

0,84

$4,68 \quad 0,57$

$4,53 \quad 0,60$

$4,45 \quad 0,67$

$4,29 \quad 1,01$

$4,38 \quad 0,78$

$\begin{array}{ll}4,41 & 0,62 \\ 4,21 & 1,00\end{array}$

$4,21 \quad 1,00$

$4,18 \quad 0,98$

$4,36 \quad 0,82$

$4,08 \quad 0,95$

$4,03 \quad 0,97$

En las Tablas 3 y 4 se observan la comparación entre los resultados obtenidos segmentando los datos según el sexo y la antigüedad de los participantes, respectivamente.

Tabla 3. Distribución de medias (y desviaciones estándar) de la importancia otorgada por los participantes a los doce roles docentes según sexo $(n=73)$.

\section{Rol docente}

Media DE Media DE

1.El docente como proveedor de información:

a. Docente en clases teóricas.

$4,03 \quad 0,86 \quad 3,95 \quad 0,83$

b. Docente en las prácticas o en la clínica.

4,75

0,50

4,63

0,62

2. El docente como modelo de rol:

a. Modelo de rol en el ámbito laboral.

b. Modelo de rol en el ámbito de la enseñanza.

$\begin{array}{llll}4,65 & 0,48 & 4,43 & 0,67 \\ 4,50 & 0,50 & 4,41 & 0,77\end{array}$

3. El docente como facilitador:

a. Mentor, asesor o tutor del estudiante.

b. Facilitador del aprendizaje.

$\begin{array}{llll}4,53 & 0,56 & 4,09 & 1,22 \\ 4,46 & 0,62 & 4,31 & 0,87\end{array}$

4. El docente como examinador:

a. Planificador o participante en las evaluaciones del estudiante.

b. Evaluador del currículo o de los planes de enseñanza.

$\begin{array}{llll}4,37 & 0,65 & 4,43 & 0,59 \\ 4,34 & 0,74 & 4,09 & 1,15\end{array}$

5. El docente como planificador:

a. Planificador del currículo.

$\begin{array}{llll}4,25 & 0,95 & 4,12 & 1,00\end{array}$

b. Organizador del curso.

$4,28 \quad 0,95 \quad 4,41 \quad 0,70$


6. El docente como desarrollador de recursos:

a. Productor de guías de estudio.

$4,21 \quad 0,87 \quad 3,97 \quad 1,01$

b. Desarrollador de materiales educativos.

$4,12 \quad 0,90 \quad 3,95 \quad 1,02$

Tabla 4. Distribución de medias (y desviaciones estándar) de la importancia otorgada por los participantes a los doce roles docentes según antigüedad $(n=73)$.

\begin{tabular}{|c|c|c|c|}
\hline Rol docente & Media & DE & Valor $\mathrm{p}$ \\
\hline \multirow{2}{*}{\multicolumn{4}{|c|}{ 1. El docente como proveedor de información: }} \\
\hline & & & \\
\hline Menos de 10 años & 3,97 & 0,77 & 0,908 \\
\hline Más de 10 años & 4,00 & 0,94 & \\
\hline \multicolumn{4}{|c|}{ b. Docente en las prácticas o en la clínica. } \\
\hline Menos de 10 años & 4,69 & 0,59 & 0,822 \\
\hline Más de 10 años & 4,66 & 0,54 & \\
\hline \multicolumn{4}{|c|}{ 2. El docente como modelo de rol: } \\
\hline \multicolumn{4}{|c|}{ a. Modelo de rol en el ámbito laboral. } \\
\hline Menos de 10 años & 4,48 & 0,63 & 0,440 \\
\hline Más de 10 años & 4,60 & 0,56 & \\
\hline \multicolumn{4}{|c|}{ b. Modelo de rol en el ámbito de la enseñanza. } \\
\hline Menos de 10 años & 4,34 & 0,71 & 0,114 \\
\hline Más de 10 años & 4,60 & 0,56 & \\
\hline \multicolumn{4}{|c|}{ 3. El docente como facilitador: } \\
\hline \multicolumn{4}{|c|}{ a. Mentor, asesor o tutor del estudiante. } \\
\hline Menos de 10 años & 4,32 & 0,80 & 0,703 \\
\hline Más de 10 años & 4,23 & 1,25 & \\
\hline \multicolumn{4}{|c|}{ b. Facilitador del aprendizaje. } \\
\hline Menos de 10 años & 4,32 & 0,80 & 0,448 \\
\hline Más de 10 años & 4,46 & 0,73 & \\
\hline \multicolumn{4}{|c|}{ 4. El docente como examinador: } \\
\hline \multicolumn{4}{|c|}{$\begin{array}{l}\text { a. Planificador o participante en las evaluaciones del } \\
\text { estudiante. }\end{array}$} \\
\hline Menos de 10 años & 4,37 & 0,61 & 0,525 \\
\hline Más de 10 años & 4,46 & 0,62 & \\
\hline \multicolumn{4}{|c|}{ b. Evaluador del currículo o de los planes de enseñanza. } \\
\hline Menos de 10 años & 4,18 & 1,02 & 0,844 \\
\hline Más de 10 años & 4,23 & 0,97 & \\
\hline \multicolumn{4}{|c|}{ 5. El docente como planificador: } \\
\hline Menos de 10 años & 4,09 & 0,99 & 0,377 \\
\hline Más de 10 años & 4,30 & 0,95 & \\
\hline \multicolumn{4}{|c|}{ b. Organizador del curso. } \\
\hline Menos de 10 años & 4,34 & 0,86 & 0,928 \\
\hline & 4,36 & 0,76 & \\
\hline \multicolumn{4}{|c|}{ 6. El docente como desarrollador de recursos: } \\
\hline \multicolumn{4}{|c|}{ a. Productor de guías de estudio. } \\
\hline Menos de 10 años & 4,06 & 1,03 & 0,895 \\
\hline Más de 10 años & 4,10 & 0,84 & \\
\hline \multicolumn{4}{|c|}{ b. Desarrollador de materiales educativos. } \\
\hline Menos de 10 años & 3,93 & 1,00 & 0,310 \\
\hline Más de 10 años & 4,16 & 0,91 & \\
\hline
\end{tabular}

En la tabla 5 se observa una comparación con los resultados obtenidos en estudio similar realizado por Nawabi y colaboradores ${ }^{(10)}$. 
Tabla 5. Comparación de resultados.

\begin{tabular}{|c|c|c|c|}
\hline Rol docente & $\begin{array}{l}\text { Media } \\
\text { Torales et } \\
\text { al. }\end{array}$ & $\begin{array}{l}\text { Media } \\
\text { Nawabi et } \\
\text { al. }\end{array}$ & Valor p \\
\hline \multicolumn{4}{|l|}{$\begin{array}{l}\text { 1. El docente como proveedor de } \\
\text { información: }\end{array}$} \\
\hline a. Docente en clases teóricas. & 3,99 & 4,2 & 0,125 \\
\hline $\begin{array}{l}\text { b. Docente en las prácticas o en la } \\
\text { clínica. }\end{array}$ & 4,68 & 4,3 & 0,002 \\
\hline \multicolumn{4}{|l|}{$\begin{array}{l}\text { Clinica. } \\
\text { 2. El docente como modelo de rol: }\end{array}$} \\
\hline a. Modelo de rol en el ámbito laboral. & 4,53 & 3,9 & $<0,001$ \\
\hline $\begin{array}{l}\text { b. Modelo de rol en el ámbito de la } \\
\text { enseñanza. } \\
\text { 3. El docente como facilitador: }\end{array}$ & 4,45 & 3,9 & 0,001 \\
\hline a. Mentor, asesor o tutor del & 4,29 & 4,1 & 0,194 \\
\hline estudiante. & 4,38 & 4,1 & 0,916 \\
\hline \multicolumn{4}{|l|}{$\begin{array}{l}\text { b. Facilitador del aprendizaje. } \\
\text { 4. El docente como examinador: }\end{array}$} \\
\hline $\begin{array}{l}\text { a. Planificador o participante en las } \\
\text { evaluaciones del estudiante. }\end{array}$ & 4,41 & 4,1 & 0,143 \\
\hline $\begin{array}{l}\text { b. Evaluador del currículo o de los } \\
\text { planes de enseñanza. } \\
\text { 5. El docente como planificador: }\end{array}$ & 4,21 & 3,9 & 0,495 \\
\hline a. Planificador del currículo. & 4,18 & 3,9 & 0,871 \\
\hline \multicolumn{4}{|l|}{$\begin{array}{l}\text { 6. El docente como desarrollador } \\
\text { de recursos: }\end{array}$} \\
\hline a. Productor de guías de estudio. & 4,08 & 3,3 & 0,645 \\
\hline $\begin{array}{l}\text { b. Desarrollador de materiales } \\
\text { educativos. }\end{array}$ & 4,03 & 3,9 & 0,821 \\
\hline
\end{tabular}

\section{DISCUSIÓN}

Grandes cambios se han producido en la educación médica en los últimos 10 años. La enseñanza integrada, el aprendizaje basado en problemas, el aprendizaje basado en la comunidad, los programas curriculares basados en competencias, la utilización de asignaturas opcionales o electivas, y el proceso de planificación curricular han sido temáticas emergentes ${ }^{(6)}$.

Para que esas temáticas se vuelvan sostenidas y que para la educación médica mejore su calidad, es necesario revisitar las actitudes de los docentes de medicina hacia sus propias técnicas y procesos de enseñanza y hacia el aprendizaje de sus estudiantes ${ }^{(9,10)}$. Los cambios de actitud necesitan, antes que nada, una autoevaluación y toma de conciencia sobre los roles que cada docente tiene y su responsabilidad, como educador, en el desarrollo de actitudes acordes a cada uno de esos roles ${ }^{(10)}$.

En el modelo de Harden y Crosby ${ }^{(7)}$, algunos de los doce roles propuestos requieren de experticia médica, mientras que otros requieren de experticia pedagógica, para poder ser cumplidos por el docente de medicina (Tabla 6).

Tabla 6. Experticia y roles en docentes de medicina.

\begin{tabular}{ll}
\hline $\begin{array}{l}\text { Tipo de experticia } \\
\text { requerida }\end{array}$ & Roles \\
\hline & -Docente en clases teóricas. \\
& -Docente en las prácticas o en la clínica. \\
Experticia médica & -Modelo de rol en el ámbito laboral. \\
& -Modelo de rol en el ámbito de la enseñanza. \\
& -Facilitador del aprendizaje. \\
& -Desarrollador de materiales educativos. \\
\hline
\end{tabular}




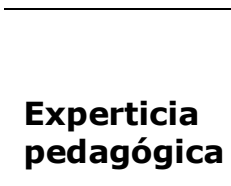

-Mentor, asesor o tutor del estudiante.

-Planificador o participante en las evaluaciones del estudiante.

-Evaluador del currículo o de los planes de enseñanza.

-Planificador del currículo.

-Organizador del curso.

-Productor de guías de estudio.

Tal como lo mencionaron Castilla Luna y López de Mesa, la mayoría de los docentes de medicina evidencia varios roles en su quehacer diario, por lo que todos deben ser potenciados por la institución educativa donde prestan servicios ${ }^{(6)}$. Este aspecto debería ser evaluado en las universidades paraguayas en próximas investigaciones.

Hassan et al. han afirmado que los docentes de medicina tienen un papel fundamental en la educación superior y que claramente pueden desempeñar un rol importante en la realización de estudios de investigación pedagógica. Dichos estudios son la base para mejorar las prácticas de enseñanza y aprendizaje de las ciencias médicas, evaluar los programas educativos y generar y probar teorías educativas. Todo lo anterior reforzará a largo plazo la calidad de las ciencias médicas ${ }^{(11)}$.

Los resultados del presente estudio nos permiten afirmar que el docente de medicina de Paraguay es más que un instructor de clases teóricas o prácticas clínicas. El docente de medicina paraguayo cumple muchos roles diferentes, todos al mismo tiempo, en consonancia con el modelo de "doce roles docentes" de Harden y Crosby ${ }^{(7)}$.

Es importante recalcar que los resultados de este estudio indican que la mayoría de los docentes de medicina paraguayos perciben que su papel como proveedor de información en clases teóricas, antes conocidas como "clases magistrales", es el menos importante. No obstante, la mayoría de los docentes percibió su papel más importante como proveedor de información en las prácticas o en la clínica y como modelo de rol en el ámbito laboral y en la enseñanza. Todo lo anterior está de acuerdo con el currículo tradicional, donde los docentes de medicina prefieren aún el "aprendizaje centrado en el docente"(10) y se consideran la principal fuente de información o inspiración del estudiante de medicina.

Este estudio evidenció que otras funciones (por ejemplo, evaluador el currículo o de los planes de enseñanza, planificador del currículo, mentor o asesor del estudiante, o productor de guías de estudio) recibieron menos preferencia que las citadas previamente. Todos estos roles forman parte de la experticia pedagógica que se espera de un docente de medicina ${ }^{(7)}$, más allá de su experticia médica.

Al comparar los resultados por sexo o por antigüedad en la docencia, no se encontraron diferencias estadísticamente significativas. Los resultados del presente estudio, al ser comparados con instituciones educativas de un país en desarrollo como Pakistán ${ }^{(10)}$, mostraron que las diferencias más significativas se encontraron en lo relativo al docente como modelo de rol en el trabajo o en el ámbito de la enseñanza. Esto podría deberse a los diferentes contextos culturales de ambos países, por lo que se necesitarían más investigaciones para explorar estos resultados y compararlos con trabajos similares realizados en países tanto de Latinoamérica como de Europa, buscando coincidencias y también diferencias relacionadas con la globalización y expansión del rol del docente de medicina ${ }^{(4,5)}$.

Es innegable que los docentes de medicina se sienten más cómodos con roles que dependen de su experticia como médico (por ejemplo, docente en la clínica o en las prácticas), y que encuentran mayores dificultades a la hora de ejercer roles que requieren formación específica en pedagogía, para los que la universidad no los ha preparado. Esto coincide con lo afirmado por Johnston ${ }^{(12)}$, que insistió que el saber disciplinario (en este caso la experticia médica) es el corazón del enfoque pedagógico de los docentes y que los mismos ejercen la docencia más por intuición y tradición que por conocimiento de los mecanismos inherentes a la misma.

Para llevar a cabo estas múltiples tareas se requiere de la profesionalización de los docentes en temas educacionales. Esto último no debería basarse sólo en el interés y la motivación de los docentes por formarse, sino que debería estar sustentado en programas formales de capacitación docente, impulsados y ofrecidos por las propias universidades ${ }^{(2,13-}$ 15). En Paraguay, sólo una de las unidades académicas incluidas en el presente estudio (la Facultad de Ciencias Médicas de la Universidad Nacional de Asunción) ha tomado acciones concretas y sistemáticas para la profesionalización de su cuerpo docente ${ }^{(16)}$. 
En 2010, directivos y docentes de la Facultad de Ciencias Médicas de la Universidad Nacional de Asunción tomaron nota de que no se contaba con un plan de desarrollo de la docencia, ni con un perfil docente que oriente la formación del médico en el ámbito de la misma; asimismo la mayoría de las acciones docentes estaban reguladas por la voluntad individual, lo que generaba desempeños heterogéneos en la docencia y una disminución del compromiso y sentido de pertenencia con la institución. Es por ello que se conformó una Comisión Local de Docencia que luego de años de trabajo logró establecer un documento que delinea el perfil genérico del docente de esta Facultad, contextualizado global y localmente, como base para generar un plan de desarrollo docente. Este plan, actualmente en vigencia, sirve como guía del itinerario formativo del docente de medicina y, además, sienta las bases para la evaluación y promoción de la carrera docente en dicha unidad académica $^{(17)}$.

Un docente de medicina no debe ser considerado solamente como un "instructor de tareas", sino como un guía que, a través de múltiples roles, ayuda al estudiante en su transformación de bachiller a médico. Si bien el modelo de Harden y Crosby proporciona suficiente comprensión de los diferentes roles que tiene un docente de medicina, se queda corto a la hora de establecer competencias específicas, necesarias para poder hace frente a sus funciones de planificadores del currículo(10), modelos de rol en el trato y manejo de pacientes $^{(18)}$, y ejemplos de capacidad comunicativa y cumplimiento de la ética médica ${ }^{(19)}$.

Jiménez et al. ${ }^{(17)}$ sostienen que la docencia médica implica la movilización y articulación dinámica y dialógica entre los saberes propios de la disciplina o profesión (la experticia médica) y los saberes pedagógicos (la experticia pedagógica). Además, a estos se suman los saberes que provienen de la experiencia, tanto del ámbito de la medicina y de la docencia como de la vida misma.

Con base en lo anterior, se considera absolutamente válida la propuesta de competencias específicas para un docente de medicina, elaboradas por la Comisión Local de Docencia de la Facultad de Ciencias Médicas de la Universidad Nacional. Estas competencias ${ }^{(17)}$, adaptadas y resumidas en la tabla 7, podrían servir de ejemplo para las otras universidades incluidas en este estudio y por qué no, para otras universidades del país o de la región latinoamericana, luego de atravesar por un proceso de validación contextual.

Tabla 7. Competencias específicas de un docente de medicina.

\begin{abstract}
Un docente de medicina articula dinámica y dialógicamente sus saberes: 1. Saberes disciplinares y/o profesionales (dominio del conjunto de disciplinas que hacen a la profesión médica o a la especialidad médica); 2. Saber pedagógico (dominio del modo de planificar, organizar y dirigir el proceso de enseñanza-aprendizaje de las ciencias médicas utilizando metodologías didácticas pertinentes, además de reflexionar sistemáticamente sobre las prácticas docentes y de investigación) y 3. Saber experiencial (resultado de la experiencia que le brinda la práctica médica, la práctica en aula, y la vida misma).
\end{abstract}

Un docente de medicina diseña y planifica los itinerarios del proceso de enseñanza-aprendizaje orientados a logros 0 resultados de aprendizaje en un currículo integrado, basado en competencias. Selecciona los contenidos con criterios de pertinencia al contexto local y global. Diseña las actividades curriculares seleccionando las metodologías didácticas pertinentes e innovadoras, incorporando las tecnologías de la comunicación e información. Diseña y aplica sistemas de evaluación coherentes con la propuesta curricular.

Un docente de medicina investiga en el ámbito de la docencia, generando así conocimientos que constituyen las bases del mejoramiento continuo en docencia. Innova y recambia constantemente las técnicas, recursos, actividades y estrategias en la docencia médica, basado en la reflexión constante sobre la praxis, la autoevaluación, los avances tecnológicos y del conocimiento.

\author{
Gestión de la \\ investigación e \\ innovación en \\ docencia
}


Además de establecer competencias específicas para la docencia, que ayuden al médico a cumplir con sus múltiples roles, es necesario que la formación del cuerpo docente de toda escuela de medicina esté inserta en los planes de desarrollo organizativo y estratégico institucional, promoviendo actividades de desarrollo de la docencia, propiciando ambientes educacionales que incentiven y reconozcan el liderazgo, la innovación y la excelencia en docencia ${ }^{(20)}$ y apoyando el proceso de desarrollo de planes curriculares acordes a los nuevos tiempos de la educación médica ${ }^{(21)}$.

Una limitación de la presente investigación es que no incluyó un test de sinceridad. Es debido a lo anterior que los participantes, al conocer los roles ideales de un buen docente, pudieron haber respondido con puntajes más elevados. Además, tampoco se incluyó un componente cualitativo, que hubiera podido dar información adicional para la triangulación de los resultados obtenidos. Otra limitación es el tipo de diseño utilizado (no probabilístico, por conveniencia) y la poca cantidad de profesores participantes.

Finalizando, se coincide con Harden y Crosby, quienes luego de identificar los doce roles de un docente de medicina, afirmaron que un buen docente es "más que un conferencista". No obstante, se debe recordar que el proceso de enseñanza-aprendizaje no es unidireccional y que los estudiantes de medicina no son niños, por lo que la relación entre un docente de medicina y sus estudiantes involucrará no solo a la pedagogía, sino también a la andragogía. En ese contexto, es de destacar a Karakitsiou et al., quienes afirmaron que un estudiante de medicina es "más que un oyente" y propusieron los " $12+1$ " roles del estudiante de medicina ${ }^{(22)}$. Estos " $12+1$ " roles del estudiante de medicina son complementarios a los doce roles del docente y pueden ayudan a remodelar la comprensión de la educación médica actual. Lo anterior requiere de más y mejores investigaciones en educación y docencia médica, a fin de reflejar aún más las complejidades propias del proceso de enseñanza-aprendizaje de todas las ciencias médicas.

\section{Agradecimientos}

Los autores expresamos nuestra gratitud al profesor Ronald Harden, de la Asociación para la Educación Médica en Europa, por autorizar gentilmente la traducción al español y la utilización de su cuestionario.

\section{Financiación}

El estudio no recibió apoyo financiero y los autores se declaran sin conflicto de intereses, siendo los mismos quienes autofinanciaron el proyecto.

\section{REFERENCIAS BIBLIOGRÁFICAS}

1. García-García JA, González Martínez JF, Estrada Aguilar L, Uriega-González Plata S. Educación médica basada en competencias. Rev Med Hosp Gen Mex 2010;73(1):57-69.

2. Montero L, Triviño $X$, Sirhan M, Moore $P$, Leiva L. Barreras para la formación en docencia de los profesores de Medicina: una aproximación cualitativa. Rev Médica Chile. 2012;140(6):695-702. Doi: 10.4067/S0034-98872012000600001

3. Ruiz LF, Pachano L. La docencia universitaria y las prácticas evaluativas. Educere 2005;9(31):531-40.

4. Álvarez-Rojo V, Asensio-Muñoz I, delFrago J, García-Lupión B, García-Nieto N, García-García M, et al. Perfiles docentes para el Espacio Europeo de Educación Superior (EEES) en el ámbito universitario español. Rev Electrónica Investig Eval Educ 2009;15(1):1-8.

5. Pérez C, Fasce $E$, Coloma K, Vaccarezza G, Ortega J. Perfeccionamiento docente desde la perspectiva de académicos de carreras de la salud de la zona centro sur de Chile. Rev Médica Chile 2013;141(6): 787-92.

6. Castilla Luna M, López de Mesa C. Los roles del docente en la educación médica. Educ Educ. 2007;10(1):105-13.

7. Harden RM, Crosby JR. The good teacher is more than a lecturer-the twelve roles of the teacher. Med Teach. 2000;22(4):334-47. Doi: $10.1080 / 014215900409429$.

8. Harden RM, Davis MH, Crosby JR. The new Dundee medical curriculum: a whole that is greater than the sum of the parts. Medical Education 1997; 31(4):264-71. Doi: $10.1111 / \mathrm{j} .1365-$ 2923.1997.tb02923.x

9. Roberts M. Producing tomorrow's doctor: the new challenge for today's undergraduate medical curriculum. J Vocat Educ Train 2004;56(4):467-84. Doi: $10.1080 / 13636820400200265$

10. Nawabi S, Khan RA, Yasmin R. Teachers' perceptions of their roles in three medical colleges in Islamabad, Pakistan. AHPE 2015;1(1):24-9. 
11. Hassan T, Bani I, Ageely H, Fauzi M. An Ideal Medical Teacher. Educ Med J 2011; 3(1): e54-e59.

12. Johnston, $\mathrm{S}$. What can we learn about teaching from our best university teachers? Teaching in Higher Education $1996 ; 1(2): 213-25$.

Doi: $10.1080 / 1356251960010204$

13. Sánchez I. La carrera académica del profesor clínico de medicina. Rev Med Chile 2009;137:1113-36. Doi: 10.4067/S0034-98872009000800018

14. Lown BA, Newman LR, Hatem CJ. The personal and professional impact of a fellowship in medical education. Acad Med. 2009;84(8):1089-97. Doi: 10.1097/ACM.0b013e3181ad1635

15. Triviño $X$, Sirhan M, Moore P, Reyes C. Formación en educación de los docentes clínicos de medicina. Rev Med Chile 2009;137:1516-22. Doi: 10.4067/S003498872009001100018

16. Comité de Autoevaluación - Modelo Nacional, Carrera de Medicina (Sede Central). Informe de Autoevaluación de la Carrera de Medicina, Facultad de Ciencia Médicas, Universidad Nacional de Asunción. Asunción: Comité de Autoevaluación; 2017.

17. Jiménez JT, Palacios $M$, Aparicio $C$, Cañete $F$, Calderoli $F$, Bogado $N$, et al. Propuesta del perfil docente basado en competencias de la Facultad de Ciencias Médicas de la Universidad Nacional de Asunción. An Fac Cien Méd (Asunción) 2016;49(1):119-40. Doi: 10.18004/anales/2016.049(01)119-140

18. Mileder LP, Schmidt A, Dimai HP. Clinicians should be aware of their responsibilities as role models: a case report on the impact of poor role modeling. Med Educ Online 2014;19:23479. Doi: 10.3402/meo.v19.23479

19. Leinster S. Medical education and the changing face of healthcare delivery. Med Teach. 2002; 24(1):13-5. Doi: 10.1080/00034980120103423

20. Steinert Y. Faculty development in the new millenium: key challenges and future directions. Med Teach 2000;22(1):44-50. Doi: $10.1080 / 01421590078814$

21. Mclean M, Gibbs T. Twelve tips to designing and implementing a learnercentred curriculum: Prevention is better than cure. Med Teach. 2010;32(3):22530. Doi: $10.3109 / 01421591003621663$

22. Karakitsiou DE, Markou A, Kyriakou $P$, Pieri M, Abuaita $M$, Bourousis $E$, et al. The good student is more than a listener - The $12+1$ roles of the medical student. Med Teach. 2012;34(1):e1-8. Doi: 10.3109/0142159X.2012.638006 